\section{Fun with Photoshop}

Tina (Weatherby) Carvalho,

Biological EM Facility, University of Hawaii

Image editing sofiware is making it possible for us as microscopists to digitally prepare our micrographs for output as working prints, figures for journals, slides, inclusion in reports, or as files for transmission via the Internet or World Wide Web. In the course of becoming familiar with various sofiware applications, I decided to have some fun colorizing and recombining some scanning electron micrographs. While this kind of frivolity has no place in the presentation of scientific data, learning to manipulate images in this silly way serves two purposes; both to bring humor into the steep learning curve, and to highlight the sobering fact that you can no longer believe what you see in print. Although we would all hate to admit it, there may be unscrupulous scientists in our midst, tempted to alter visual data to suit their needs. Having said that, let me now show you one way it can be done...!

First, open Photoshop 3.0 or 4.0 . I am presuming that you know your way around Photoshop, somewhat. Open each of the image files you will use for the composite picture (i.e., Figure 1).

Prepare the image by cropping, rotating, or flipping, as necessary. Adjust the levels and contrast and brightness as needed (covered in next month's article). Paint your images as your imagination dictates. Experiment; different tools give different results. Be creative (i.e., Figure 2)! Save your images with new names along the way (Save As...) so that you can go back to previous versions, if desired.

Now you need to select the portion of one image that you want to move onto the other. Selections can be made in several ways; with the magic wand or with Select $\rightarrow$ Color Range, both of which are selected by color; by tracing with the lasso tool; or by using the paths tool which can give smoother curves if you are already familiar with it from Adobe Illustrator. Remember to double-click on the tool to show the options palette to help refine your tool.

Afier you have made your selection, click on the Move Tool and drag the selection to the destination image. Another option is to copy the selection to the clipboard with Edit $\rightarrow$ Copy, then click on the destination image to activate it and use Edit $\rightarrow$ Paste to bring over the selection from the clipboard. This is now a floating selection, and can be dragged around with the Move tool. Position it where you want it, then either paste it down (if you are sure) by deselecting it, or keep it as its own layer by dragging the floating selection to the little folded paper icon at the bottom of the Layers palette in 3.0. It should remain as its own layer in 4.0. As a layer it can be further moved and manipulated. Be sure the Preserve Transparency box remains checked in the Layers palette. Each layer or the background can be turned off by clicking on the eyeball next to it in the palette, or layers can be thrown away by dragging to the trash can at the bottom of the Layers palette.

Was it the wrong size? Resize the selection with Image $\rightarrow$ Image Size (i.e., Figure 3). Experiment with Image $\rightarrow$ Flip, Image $\rightarrow$ Rotate, and Image $\rightarrow$ Effects $\rightarrow$ Scale, Skew, Perspective, or Distort. You can further refine the illusion by adding shadows and adjusting lighting angles. Photoshop 4.0 comes with quite a few special effects, which are also available as plug-ins for Photoshop 3.0.

When the image is as you like it, save it, either in the .psd Photoshop format, which preserves the layers, or Flatten it by clicking on the right-pointing arrow in the Layers palette and selecting Flatten from the popout menu, then Save as a TIFF or JPEG file.

Here is an example of an image on which I spent a little more time (i.e., Figure 4). In the original image there were two girls sitting in front of my Hitachi S-800 FESEM, with an insect on the CRTs. For the final image I had to completely remove one child, move her chair, clone parts of the SEM stage (giving it more knobs and meters than it really has), add the pseudocolored insect (a native Hawailan bug), give him a shadow, and put an image of my daughter on the screens of the SEM. The whole effort took far more time than l'd like to admit, and a lot of RAM.

In future articles I will address techniques of legitimate interest to microscopists. I have prepared a step-by-step tutorial designed to get you through the very basics of opening an image file, making corrections to it, adding arrows, labels, and a magnification bar, followed by page layout, all with Photoshop. In an effort to provide more detail, I am working on a manuscript for a handbook tentatively titled "Photoshop Distilled: Tips on Black and White Image Adjustment and Layout for Scientists". I would appreciate any comments, criticisms, or contributions! I can be reached by e-mail at: tina@pbrc.hawaii.edu.

For more examples of pseudocolored SEMs, visit my web site at http://www.pbrc.hawaii.edu/bemf/microangela.

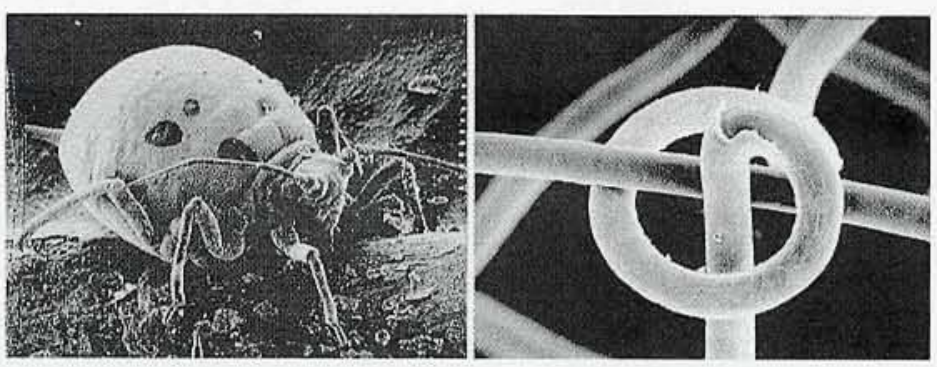

Figure 1

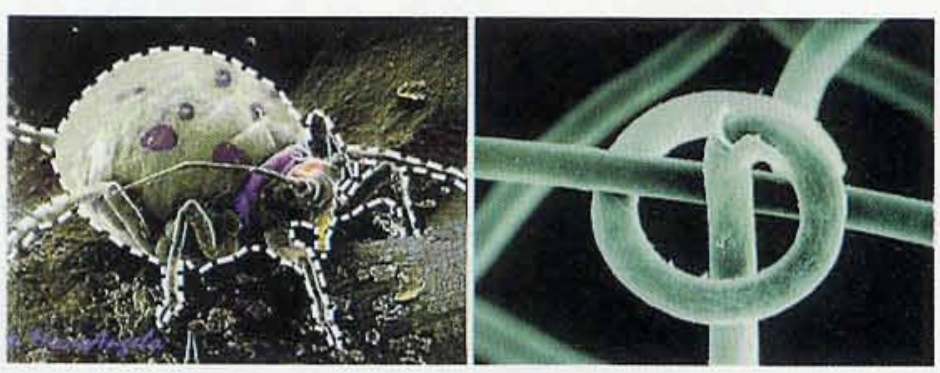

Figure 2
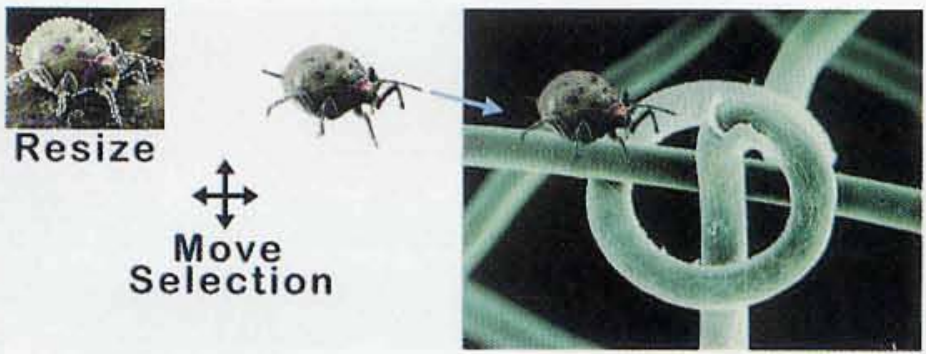

Figure 3

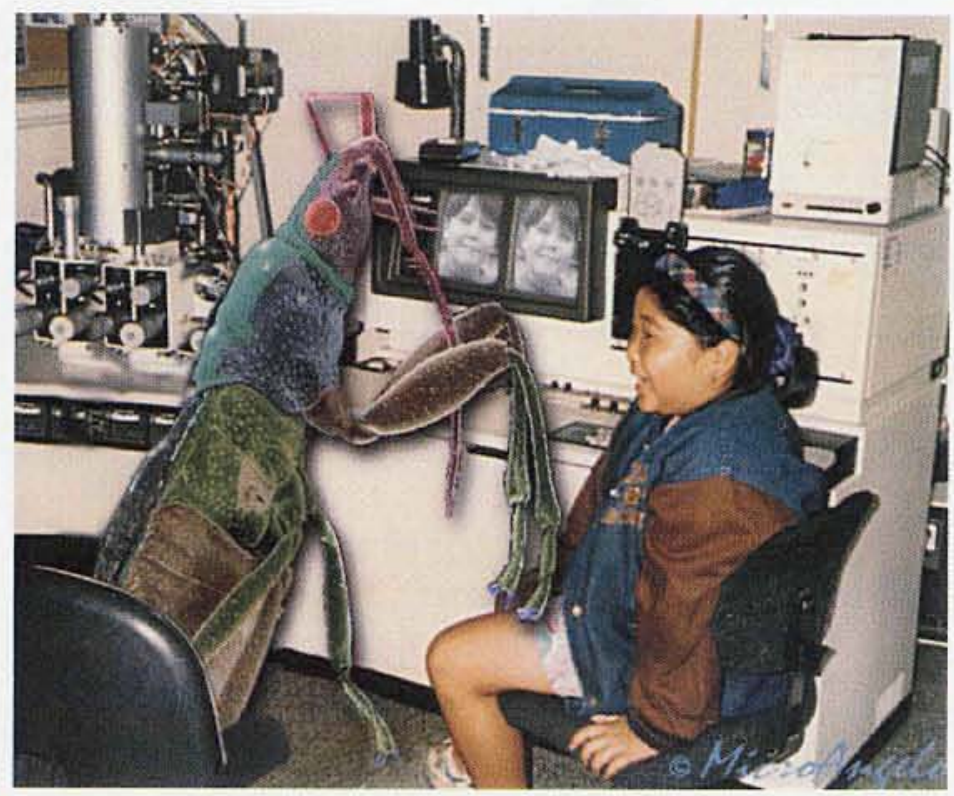

Figure 4 


\section{Ready for a change
of environment?}

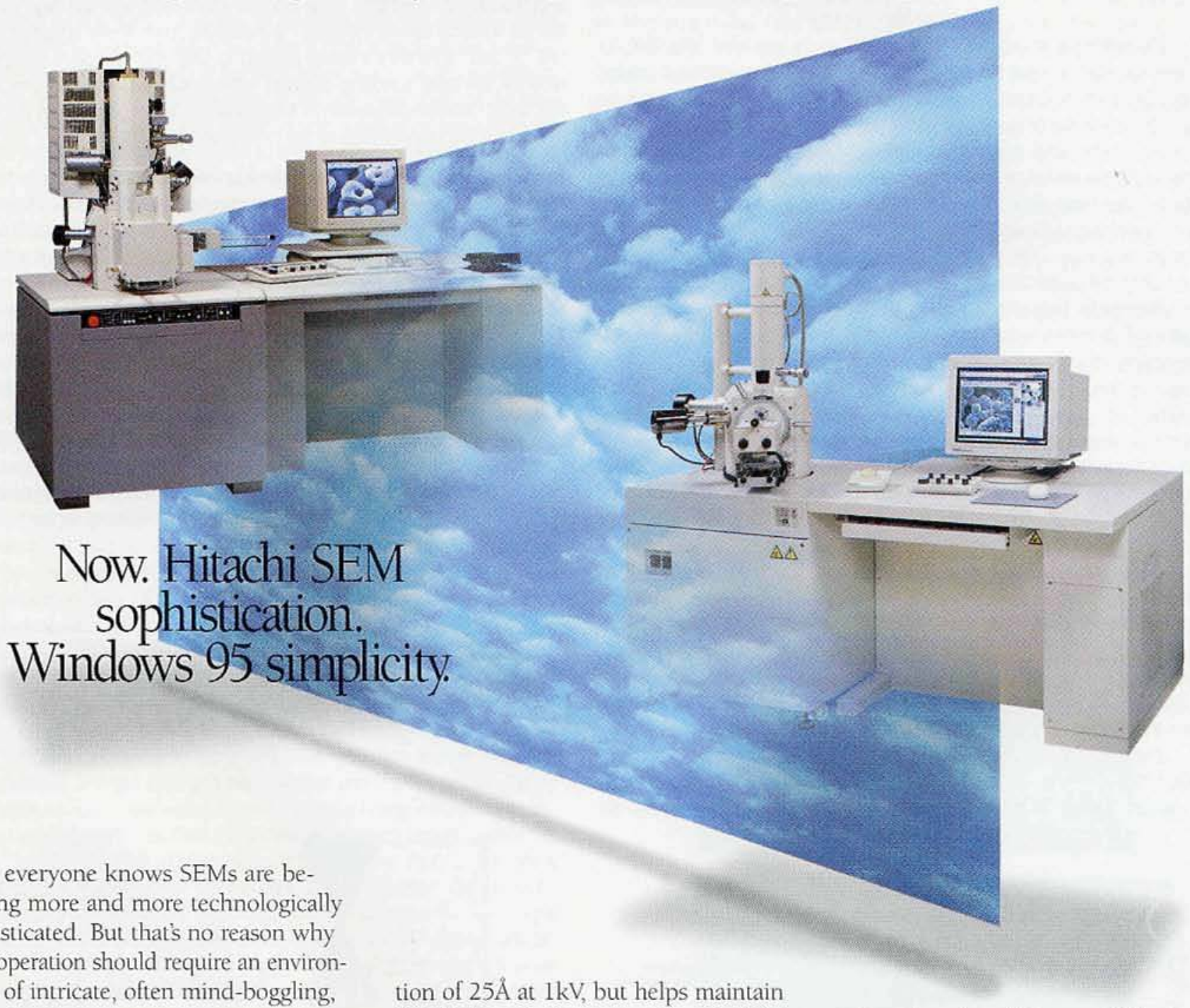

Sure, everyone knows SEMs are becoming more and more technologically sophisticated. But that's no reason why their operation should require an environment of intricate, often mind-boggling, challenges.

Enter Hitachi's new S-4700 and S-3500N SEMs, which replace all that operational complexity with the easy familiarity of Windows ${ }^{\mathrm{TM}} 95$ control. So anyone who's ever used a Windowsbased PC can - with a near-zero learning curve - take over their operation. And how's this? To keep die-hard traditionalists just as excited, these SEMs can come set up for convenient switching to conventional control as well.

Our S-4700 FE SEM and S-3500N Variable Pressure SEM offer even more to be excited about. Like superior resolution. The S-4700's new objective lens, for example, not only contributes to a resolu- that high resolution even when studying large samples at high tilt angles and at long working distances. Further, both instruments are available with our $\mathrm{Hi}$ Mouse feature, for operating EDX systems right from the SEM. (In fact, the S-4700's analytical mode permits EDX operation concurrent with high-resolution imaging.) And, with our Rapid Image Shift Movement (RISM) capability, you'll have the fastest, most precise specimen navigation ever.

So. Super performance made super easy. It's surely a refreshing environment for SEM microscopy. And just as surely, you'll want details on these breathrough SEMs. You can get them easily by phone or E-mail, or by visiting our Web sitewhich, of course, you'll very likely do via a friendly Windows environment.

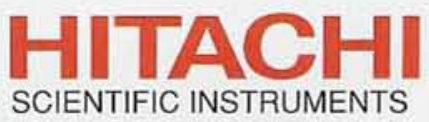

Nissei Sangyo America, Ltd.

755 Ravendale Drive

Mountain View, CA 94043 (800) 227-8877

E-mail: sidsales@nissei.com www.nissei.com

25 West Watkins Mill Road Gaithersburg, MD 20878 (800) 638-4087

Windows is a registered trademark of Microsolt Corporation 